\title{
Tackling Social Cognition in Schizophrenia: A Randomized Feasibility Trial
}

\author{
Rumina Taylor, Matteo Cella, Emese Csipke, Charles Heriot-Maitland, Caroline Gibbs \\ and Til Wykes
}

Institute of Psychiatry, Psychology and Neuroscience, King's College London, UK

\begin{abstract}
Background: Social cognition difficulties in schizophrenia are seen as a barrier to recovery. Intervention tackling problems in this domain have the potential to facilitate functioning and recovery. Social Cognition and Interaction Training (SCIT) is a manual-based psychological therapy designed to improve social functioning in schizophrenia. Aims: The aim of this study is to evaluate the feasibility and acceptability of a modified version of SCIT for inpatient forensic wards. The potential benefits of the intervention were also assessed. Method: This study is a randomized single blind controlled design, with participants randomized to receive SCIT $(N=21)$ or treatment as usual (TAU; $N=15$ ). SCIT consisted of 8 -week therapy sessions twice per week. Participants were assessed at week 0 and one week after the intervention on measures of social cognition. Feasibility was assessed through group attendance and attrition. Participant acceptability and outcome was evaluated through postgroup satisfaction and achievement of social goals. Results: The intervention was well received by all participants and the majority reported their confidence improved. The SCIT group showed a significant improvement in facial affect recognition compared to TAU. Almost all participants agreed they had achieved their social goal as a result of the intervention. Conclusions: It is feasible to deliver SCIT in a forensic ward setting; however, some adaptation to the protocol may need to be considered in order to accommodate for the reduced social contact within forensic wards. Practice of social cognition skills in real life may be necessary to achieve benefits to theory of mind and attributional style.
\end{abstract}

Keywords: Social cognition, schizophrenia, social functioning, forensic, inpatient.

\section{Introduction}

Social inclusion for people with psychosis is a key priority for mental health services. Social integration acts as a protective factor and has been linked to improved wellbeing and quality of life, as well as being identified as central to recovery (Tew, Ramon, Slade, Bird and Le Boutillier, 2012). Many people with psychosis report feeling lonely and a need for more friends (Stain et al., 2012). A recent study found activity levels for people with psychosis to be compromised by fears of what others may think of them (Moriarty, Jolley,

Reprint requests to Rumina Taylor, Clinical Psychologist, Department of Psychology PO 77, Henry Wellcome Building, Institute of Psychiatry, Psychology and Neuroscience, King's College London, De Crespigny Park, London SE5 8AF, UK. E-mail: rumina.taylor@kcl.ac.uk 
Callanan and Garety, 2012). Despite its recognized importance as a prognostic factor, current mainstream interventions have achieved very limited results. The first line of treatment for schizophrenia, antipsychotic medication, has shown negligible effects on social impairment (Combs et al., 2009). Psychosocial interventions are considered more appropriate to tackle social functioning problems but social skills training has not been used frequently in the UK and is not recommended by national guidelines (e.g. National Institute for Health and Care Excellence, NICE, 2014).

There are two intervention approaches to target social cognitive impairments in people with psychosis. The first integrates social cognitive training within other therapy packages. Roder, Mueller and Schmidt (2011), for example, have combined cognitive remediation with social skills therapy. Their Integrated Psychological Therapy (IPT) is a group programme consisting of neurocognitive intervention with communication, social skills training and problem-solving for those with schizophrenia. A recent review showed those who completed IPT experienced greater improvements in neurocognition, social cognition, functioning and negative symptoms when compared to a control group after the intervention and at follow-up (Roder et al., 2011). Crucially, these interventions tend to last several months and are designed for community dwelling service users. The second group of interventions targets social cognition difficulties only. One such intervention is Social Cognition and Interaction Training (SCIT) developed by Penn, Roberts, Combs and Sterne (2007). SCIT is a 24-session manual-based group treatment, including elements of cognitive behavioural therapy and social skills training. It is designed for those with schizophrenia spectrum disorders to improve social functioning by enhancing social cognition. Social cognition, which includes emotion perception (recognizing the emotions of others), theory of mind (ToM; the ability to understand another person's intentions), and attributional style (the explanations a person forms to explain the causes of positive and negative situations; Penn et al., 2005), has been identified as a treatment target to improve social functioning in schizophrenia (Roberts, Penn, Labate, Margolis and Sterne, 2010). Social cognitive abilities are impaired in people with schizophrenia (Green, Oliver, Crawley, Penn and Silverstein, 2005) and are associated with social and functional outcomes (Couture, Penn and Roberts, 2006). SCIT also targets underlying processes such as cognitive inflexibility, jumping to conclusions, and intolerance of ambiguity typically observed in people with schizophrenia that may be difficult to access using behavioural interventions (Penn et al., 2007).

Across different studies and research groups, SCIT has been shown to improve emotion perception, ToM, cognitive flexibility, tolerance of ambiguity and need for closure (Combs, Adams et al., 2007; Parker, Foley, Walker and Dark, 2013; Penn et al., 2005; Penn et al., 2007; Roberts et al., 2010; Roberts, Kleinlein and Stevens, 2012). Importantly, SCIT has been evaluated positively by participants and clinicians (Parker et al., 2013; Penn et al., 2007; Roberts et al., 2010, 2012) and social cognition improvements have been shown to generalize to better self-reported social relationships and fewer aggressive incidents within an inpatient setting with effects being maintained at 6-month follow-up (Combs et al., 2009). However, the intervention is long and many of these studies have been conducted outside the United Kingdom (UK) given the UK's lack of support for social skills training. Some studies have been additionally limited by small samples and uncontrolled pre-post designs.

The present study aims to evaluate primarily the feasibility and acceptability of an adapted, novel, and shorter version of SCIT (to facilitate implementation within a National Health Service (NHS) setting) for the first time in the UK within forensic wards. We also assessed 
any potential benefits of the intervention as secondary outcomes to inform power calculation for future larger studies. The recently published Schizophrenia Commission (2012), which examined the provision of care for people with psychosis in England, found inpatient settings to be "anti-therapeutic" with medication being prioritized over psychological interventions, despite service users requesting such treatments. Activities on wards and a therapeutic environment are important to raise the quality and outcomes of psychiatric inpatient care. We therefore hoped that gathering more evidence on the feasibility and effectiveness of a pragmatic version of SCIT would improve access to psychological therapy for inpatients and that this intervention at this early stage would promote recovery. Indeed, social cognition training has been found to facilitate recovery in schizophrenia beyond symptom stabilization as it addresses a key component of recovery, successful social functioning, by improving social cognitive abilities, which then guide effective social behaviour (Kern, Glynn, Horan and Marder, 2009). Social cognition has been shown to mediate between cognition and functional outcome (Addington, Saeedi and Addington, 2006) and is therefore a more relevant target for intervention to achieve gains in social functioning (Kern et al., 2009). Developing social cognitive therapies is thus worthwhile in order to promote recovery.

This study differs from the previous inpatient study (Combs, Adams et al., 2007) by recruiting from a pool of eligible patients rather than from those who have already shown an interest. We have also reduced the time for therapy from 18 weeks to 8 weeks, which is more suitable for shorter patient stays in an NHS environment. We hypothesized that those who completed SCIT would show greater improvement in emotion perception, ToM, and cognitive flexibility and greater improvement in hostile attributional biases for ambiguous situations as found in the previous study (Combs, Adams et al., 2007). Lastly, we expected SCIT would be positively received by participants and that they would meet their social goals or feel they were able to meet these goals as a result of the intervention to provide some indication of the functional significance of therapy. We did not measure aggressive incidents as due to the high level of risk management on the wards included such incidents were negligible. Moreover, due to the limited opportunity within such inpatient settings for performing everyday social skills, we did not formally assess social functioning on global scales as there were no opportunities for their assessment.

\section{Method}

\section{Design and procedure}

This study is a cluster randomized single blind controlled trial with a stepped wedge design. Four adult male forensic inpatient wards took part, with two wards randomized to receive SCIT and the remaining two wards received treatment as usual (TAU) in phase one. In phase two, the wards receiving TAU in phase one were offered SCIT. All participants were assessed twice: at intake and after the intervention. Participant demographic information, symptom, and social cognition effectiveness measures were administered by research assistants blind to treatment group. SCIT therapists administered measures of goal achievement and also requested completion of intervention satisfaction questionnaires although these were completed independently by participants. Ethical approval was granted by the Bexley and Greenwich Research Ethics Committee (ref 07/H0809/45). 


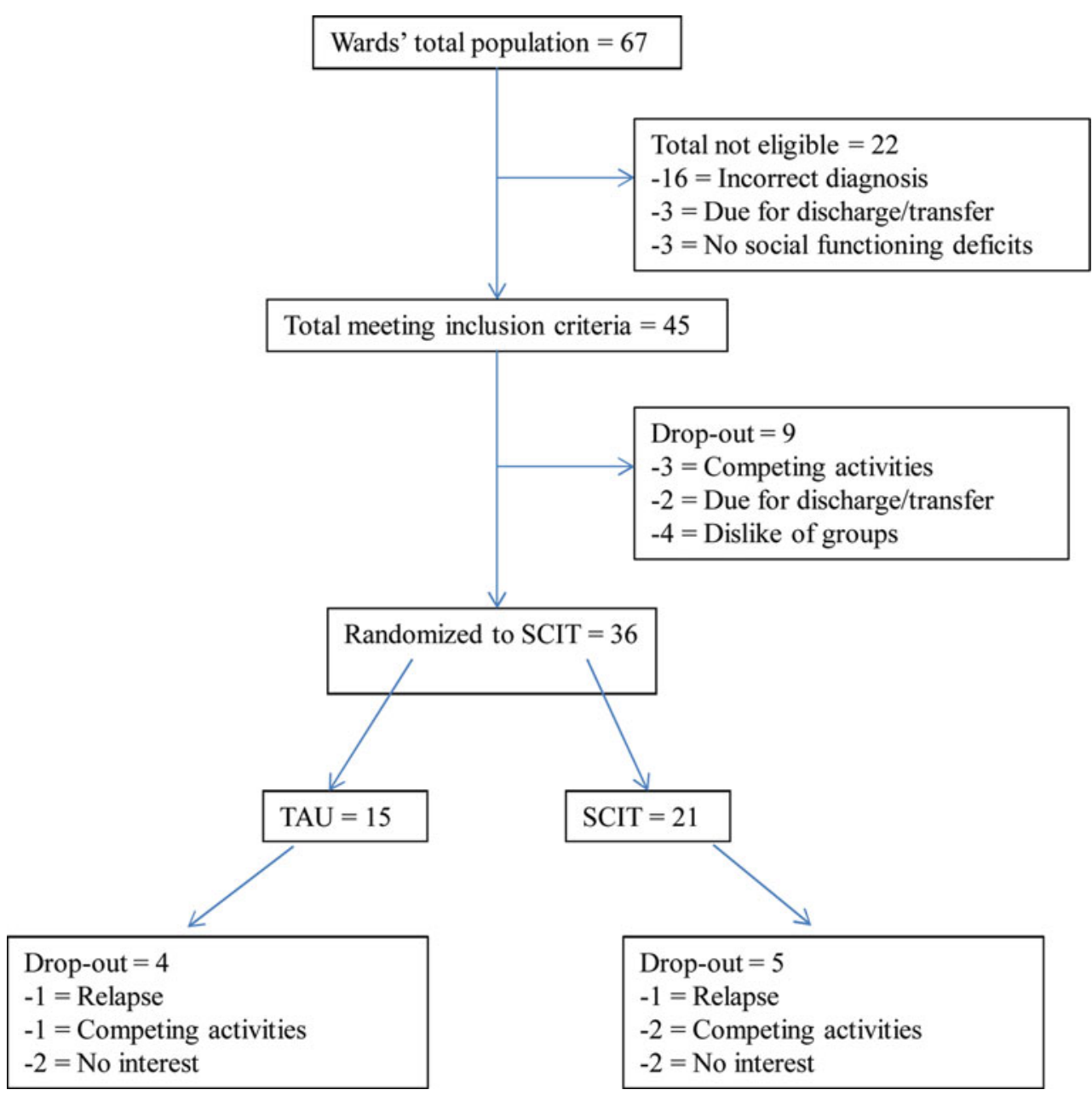

Figure 1. (Colour online) CONSORT diagram showing the flow of participants through each stage of the study

\section{Participants $(N=36)$}

Participants were recruited from secure forensic psychiatric wards in collaboration with a single inner city London NHS trust. Inclusion criteria were: aged 18-65, sufficient English to consent to the research, complete assessments and engage with the intervention, a schizophrenia spectrum diagnosis as determined by clinical teams and case notes, and difficulties with social functioning either self-reported or indicated by clinical teams. Exclusion criteria included evidence from case notes or a formal diagnosis of intellectual disability, significant substance misuse, other comorbid diagnoses, or other difficulties that would impair engagement in the group (e.g. high medication dosage impairing attention or continuous violent or aggressive behaviour). See Figure 1. 


\section{Intervention}

For the purpose of this study we adapted the original SCIT intervention, in consultation with the developers, to facilitate implementation within a NHS inpatient setting. This modified intervention was shorter and consisted of a 16-session group comprised of the same three phases as the original package. Emotion perception was the focus of the first six sessions and the following seven sessions were assigned to ToM, attributional style, and underlying processes such as jumping to conclusions and tolerating ambiguity. Integration that focuses on generalizing skills to participants' real life social situations was covered in the final two. All sessions end with the setting of homework that is reviewed at the beginning of the following session. We felt it necessary to facilitate bi-weekly shorter sessions (45 minutes) to encourage and maintain participant rapport and engagement. We also anticipated participants would have difficulty sharing their real life social situations during the integration phase so we used material from the manual to support these sessions.

\section{Measures}

Background: demographic and clinical information. Gender, age, ethnicity, marital status, accommodation status, education, current employment, time since first contact with psychiatric services, and recent clinical service use information were collected by self-report and confirmed with case notes.

Symptoms. The Positive and Negative Syndrome Scale (PANSS; Kay, Fiszbein and Opler, 1987) is a structured clinical interview consisting of 30 -items that assess the presence and severity of positive and negative symptoms associated with schizophrenia, as well as general psychopathology over the last week. Researchers were trained in how to conduct the interview and apply the ratings. Practice interviews were recorded and listened to by an expert interviewer and researchers had to be approved by the expert before carrying out research interviews.

\section{Main outcomes}

Feasibility and acceptability. Participant group attendance and attrition was recorded. Participants were asked to identify one social goal they would like to achieve or work towards during SCIT. Group facilitators ensured specific, realistic, and achievable goals within the treatment time frame and inpatient context e.g. to be able to talk to another patient on the ward about football. At the end of the intervention participants rated on a 5-point Likert scale their goal achievement, use of group skills, and confidence to give an indication of the functional significance of SCIT. All participants completed a satisfaction questionnaire (Gledhill, Lobban and Sellwood, 1998) at the end of treatment.

\section{Effectiveness}

Social cognition: emotion perception. This was measured using the Face Emotion Identification Task (FEIT; Kerr and Neale, 1993) in which participants are presented with photographs of faces and have to identify which emotion is being shown. A total score of 
correct answers is recorded. The FEIT has good psychometric properties (Kerr and Neale, 1993).

Theory of Mind (ToM). The Hinting Task (Corcoran, Mercer and Frith, 1995) was used. Ten individual short stories describing an interaction between two people are read to the participant, with each ending with one of the characters dropping a hint. Participants are required to identify what the person really meant. Scores for the task range from 0 to 20 . The Hinting Task has been used in a number of studies investigating ToM in schizophrenia and has been found to have good psychometric properties with acceptable test-retest reliability (Roberts, 2007).

Attributional style. The Ambiguous Intentions Hostility Questionnaire (AIHQ; Combs, Penn, Wicher and Waldheter, 2007) consists of 15 vignettes describing negative interpersonal situations that vary in intentionality. In line with previous research only the five items from the ambiguous scale were used as these are more sensitive to social cognitive biases (Combs, Adams et al., 2007). Responses are rated on a 0 to 5 Likert scale with higher scores indicating a higher attributional bias. In this study the questionnaire was scored by two researchers blind to treatment condition who achieved high intraclass correlation coefficients with an independent rater $(r=0.77 ; r=0.97 ; p<0.01)$ for the hostility and aggression subscales.

Cognitive insight and need for closure. These domains were assessed using the Beck Cognitive Insight Scale (BCIS; Beck, Baruch, Balter, Steer and Warman, 2004), a 15item self-report questionnaire designed to evaluate self-reflectiveness and overconfidence in interpretations of anomalous experiences with overall poorer cognitive insight indicated by lower scores. The BCIS has been shown to have strong test-retest reliability (Martin, Warman and Lysaker, 2010).

Pinkham and colleagues (2014) have recently worked with experts within the field of social cognition to identify important domains of social cognition to be measured in schizophrenia in addition to clarifying the best existing measures. Two expert surveys identified and supported the value of four domains - emotion processing, social perception, theory of mind, and attributional style, which were assessed in this study. Best measures included the AIHQ and Hinting Task which were also used in this study.

\section{Analysis}

Data integrity. Frequencies and distributions were inspected. Preliminary data analyses (i.e. ANOVAs) were conducted to assess the potential differences between participants excluded, dropped-out and those included. Differences between wards were assessed at baseline and potential differences controlled for in subsequent analysis. Practice effect for wards randomized to TAU first and subsequently receiving SCIT was assessed by comparing SCIT baseline assessment to the assessment conducted after the TAU period using $t$-test.

Analyses were conducted using SPSS version 21. All tests were two-tailed and significance level was set at $p<.05$.

Feasibility. Participant group attendance and attrition was recorded. Prior to commencing the intervention, group facilitators agreed completion of $75 \%$ of sessions was necessary to evaluate intervention benefit appropriately. This decision was based on previous clinical experience with SCIT and discussions with the original authors. Therefore, those who 
Table 1. Demographic and clinical characteristics

\begin{tabular}{llll}
\hline & SCIT $(N=21)$ & TAU $(N=15)$ & \\
& Mean $(S D)(\%)$ & Mean $(S D)(\%)$ & $\mathrm{F}(p)$ \\
\hline Age & $40.7(10.3)$ & $39.2(10.6)$ & $0.1(0.7)$ \\
Age first admission & $25.2(4.9)$ & $22.3(8.1)$ & $1.8(0.2)$ \\
Education (years) & $9.8(2.5)$ & $10.6(2.1)$ & $0.9(0.3)$ \\
Ethnicity & & & \\
Afro-Caribbean & $81 \%$ & $73 \%$ & $\mathrm{X} 2=5.3(0.32)$ \\
Other & $19 \%$ & $10.8(4.9)$ & $0.1(0.7)$ \\
PANSS positive & $10.3(2.8)$ & $10.4(3.1)$ & $0.4(0.5)$ \\
PANSS negative & $9.7(2)$ & $23.4(7.5)$ & $0.4(0.8)$ \\
PANSS general & $24(7.1)$ & & \\
\hline
\end{tabular}

completed fewer than 12 sessions of SCIT were excluded from analyses of intervention benefit and acceptability but not feasibility.

Intervention benefit. Between group differences were assessed using univariate mixedfactor ANCOVA for each outcome. For this analysis posttreatment scores were used as dependent variables, treatment as a fixed factor, and baseline scores as covariates. Treatment effects were estimated using partial eta squared $\left(\eta^{2}\right)$.

The percentages of participants who were able to achieve their social goal, or felt they could now achieve this goal following SCIT, were calculated. The percentage of participants who self-reported that their confidence had increased following the intervention was also calculated.

Acceptability. All participants completed a satisfaction questionnaire at the end of treatment. Each item was reviewed and percentages calculated to give an indication of whether clients enjoyed the group, found it beneficial, and would participate in the intervention again.

\section{Results}

\section{Participants}

During the recruitment period of this study 67 people were inpatients in the four wards considered. Of these, 45 were eligible for the study and 36 gave written informed consent to take part. Reasons for refusing participation included having competing activities that clashed with group sessions, an upcoming discharge or transfer that meant participants could not commit to all sessions, and a dislike of group interventions.

Of the 36 participants that entered the study, 15 received TAU and 21 SCIT. Table 1 shows the demographic and clinical baseline characteristics of participants in the study. There were no differences in demographics and most clinical variables across the four psychiatric wards considered. Since there were differences in the level of negative symptoms assessed, it was included as a covariate in analyses of group differences. Analysis conducted to assess the potential confounding effect on practice in the wards first randomized to TAU did not reveal any significant effects of repeated measurement. Similarly, no differences were found 
Table 2. ANCOVA's controlling for baseline and PANSS negative

\begin{tabular}{|c|c|c|c|c|c|c|}
\hline & $\begin{array}{l}\text { Pretreatment } \\
\text { SCIT }(N=21) \\
\text { Mean }(S D)\end{array}$ & $\begin{array}{l}\text { Posttreatment } \\
\text { SCIT }(N=16) \\
\text { Mean }(S D)\end{array}$ & $\begin{array}{l}\text { Pretreatment } \\
\text { TAU }(N=15) \\
\text { Mean }(S D)\end{array}$ & $\begin{array}{l}\text { Posttreatment } \\
\text { TAU }(N=11) \\
\text { Mean }(S D)\end{array}$ & $\begin{array}{l}F \text {-test } \\
\text { Group } \\
\text { effect }\end{array}$ & $\begin{array}{l}\text { Effect size } \\
\text { Between } \\
\text { group }\left(\eta^{2}\right)\end{array}$ \\
\hline $\begin{array}{l}\text { AIHQ hostility } \\
\text { scale }\end{array}$ & $1.41(.55)$ & $1.5(.76)$ & $1.61(.69)$ & $1.4(.56)$ & .41 & .06 \\
\hline $\begin{array}{l}\text { Facial affect } \\
\text { recognition }\end{array}$ & $8.69(4.5)$ & $11.06(3.1)$ & 9.45 (3.7) & $8.55(3.3)$ & $7.5^{* *}$ & .24 \\
\hline Hinting task & $13.44(4.1)$ & $14.3(2.2)$ & $13.9(2.8)$ & $14.8(2.6)$ & 0.18 & .008 \\
\hline $\begin{array}{l}\text { Beck } \\
\text { self-certainty } \\
\text { scale }\end{array}$ & $5.63(6.2)$ & $4.94(4.9)$ & $3.6(4.1)$ & $5.3(5.2)$ & 1.3 & .053 \\
\hline
\end{tabular}

${ }^{*} p<.05$

${ }^{* *} p<.001$

*** $p<.0001$

between participants who took part in the study and those who dropped-out. The majority of participants were of African-Caribbean origin, which is reflective of the population served by the trust within inner London.

\section{Feasibility}

Attrition and attendance. Of the 36 participants that completed baseline assessments, 4 from TAU and 5 from SCIT did not complete the second assessment. Reasons for noncompletion were: relapse $(N=2)$, conflict with other clinical activities $(N=3)$, and no interest $(N=4)$, with no differences between the groups. SCIT participants attended a mean of 14.4 sessions $(S D=1.4)$ out of a total of 16 sessions, an average attendance rate of $90 \%$.

Intervention benefit. Table 2 summarizes the descriptive data and the ANCOVA results for the social cognition domains and symptom dimensions considered. Participants in the SCIT group significantly improved on facial affect recognition compared to participants in the TAU group. No other effect of the intervention was found on any of the other measures used.

\section{Participant social goal achievement and confidence}

Fourteen participants completed ratings of their goal attainment and 93\% agreed (strongly or partly) that they had achieved their goal. Examples of personal goals were: "to be able to talk to another two people on the ward at lunchtime" and "to be able to visit my hostel once a week and get along with the other resident next door to me by starting a conversation". Similarly, $78 \%$ agreed (strongly or partly) they could now achieve other social goals as a result of skills acquired during SCIT. With regards to participant confidence, $92 \%$ agreed (strongly or partly) their confidence had improved following the intervention. 


\section{Therapy satisfaction}

Eighteen participants completed the satisfaction questionnaire and all stated they had enjoyed the group and found it beneficial, with $83 \%$ stating they would like to be involved in a similar group in the future. Some participants $(22 \%)$ fed back that they would have preferred to have engaged in individual work and $17 \%$ reported finding it difficult to discuss their problems in the presence of others. Many (94\%) stated they benefited from sharing specific experiences with other group members who had similar difficulties and only $11 \%$ indicated there were components of the group they did not like.

\section{Discussion}

In terms of feasibility, the adapted SCIT group seems feasible to the majority of inpatients with 16 of 21 completing at least 12 of the 16 SCIT sessions. Amongst those completers there was an attendance rate of $90 \%$, which was similar to a previous study implementing SCIT within an inpatient forensic setting (Combs, Adams et al., 2007), and higher than studies of SCIT in the community (Parker et al., 2013; Roberts et al., 2010). The intervention drop-out rate of $24 \%$ is similar to previous community-based SCIT groups (Roberts et al., 2010) but is high in comparison to previous inpatient SCIT groups (Combs, Adams et al., 2007). The difference may be due to differing recruitment processes; i.e. Combs, Adams et al. (2007) recruited from a pool of inpatients that had already shown an interest in attending, whereas in the current study, participants were recruited from a pool of eligible inpatients with no prior indication of interest.

In terms of changes in the social cognition measures, we found an improvement in emotion recognition in the SCIT group compared to TAU. However, ToM and the biased attribution measures remained comparable. The findings from the FEIT are in line with previous SCIT studies (Combs, Adams et al., 2007; Roberts et al., 2010). Perceiving and understanding emotions is the primary focus of the first six sessions of SCIT, and this skill is revisited throughout the programme in the form of "checking in" with participants' emotional awareness at the start of each session, and by repeatedly displaying an "emotion poster" that shows faces expressing different emotions. The larger focus on emotion recognition and the educational approach around this competence might be key to producing a positive effect in this domain.

In terms of changes to ToM or attribution style, they may require longer therapy and more exposure to social situations. Improvements in these two domains following social cognition training are not as common as for emotion recognition. In previous research, there have been mixed findings for ToM, with some SCIT studies showing improvements (Combs, Adams et al., 2007; Penn et al., 2005; Roberts et al., 2010) and others showing none (Horan et al., 2009; Roberts and Penn, 2009). There is a similar picture with previous findings on attributional style; e.g. Roberts et al. (2010) observed no change in AIHQ after 20 sessions of SCIT in a community sample. The non-significant finding in attribution bias in our results might also have been influenced by the already low AIHQ ratings at baseline, despite other outcome measure scores being comparable to previous studies (e.g. Combs, Adams et al., 2007; Roberts et al., 2010). This may be a consequence of the therapeutic focus of these forensic wards which targets aggressive and violent behaviour. 
The perceived usefulness and acceptability of SCIT to participants was positive. Subjective measures of perceived goal achievement showed that $93 \%$ agreed they had achieved a personal social goal set in the first session. The subjective feedback showed that participants not only found SCIT helpful in reaching these personal goals, but also that it was generally enjoyable and beneficial, and the sort of group they would like to be offered again. The perceived usefulness of the group to the participants may also be considered as having achieved an increased level of awareness towards social problems. People with schizophrenia generally have poor awareness of their symptoms; increasing awareness of social cognitive problems may be a worthwhile outcome that future research in the field of social cognition may consider (Cella, Swan, Medin, Reeder and Wykes, 2013).

A number of limitations to this study need to be noted. The sample size was small but the aim of this study was primarily to investigate the acceptability and feasibility of implementing SCIT within an inpatient setting and increase access to such interventions for this neglected group. We are somewhat limited in the recommendations we can make with regard to the effectiveness of SCIT in the study as this pilot was concerned with feasibility and acceptability. We did examine the potential benefits of SCIT but cannot at this stage draw firm conclusions about effectiveness, as larger powered studies are first required. The confined environment of an inpatient ward presented very few opportunities for practising/applying social skills. This is not so much a limitation of the study but more a recognition of the reality of providing interventions in this challenging inpatient setting. SCIT session content could be more specifically adapted to the immediate social environment, e.g. considering ward-based scenarios and setting tasks that can be carried out on the ward, rather than in the outside world. Lastly, although effectiveness assessments were administered by researchers blind to treatment group, therapists administered measures of goal achievement and group satisfaction that may have biased responses.

Moving forward, this study needs replication with a larger sample size and it would be useful to follow-up participants in the community once they have been discharged from an inpatient setting to see if the intervention impacts functioning and recovery. More formal measures of social functioning would also be a useful addition.

In conclusion, this study has extended previous research in a new setting with an adapted and much shorter programme that may be more suitable to an inpatient environment. It has shown that SCIT is a feasible and acceptable group-based intervention for NHS forensic inpatients. There are promising indications for the effectiveness of SCIT in improving emotion perception in this setting, which is an important domain of social cognition.

\section{Acknowledgements}

Financial support: This article presents independent research funded by the National Institute for Health Research (NIHR) under its Programme Grants for Applied Research scheme (RPPG-0606-1050). The views expressed in this publication are those of the authors and not necessarily those of the NHS, the NIHR or the Department of Health.

TW would like to acknowledge the support of the NIHR Biomedical Research Centre in Mental Health at the South London and Maudsley NHS Foundation Trust and the Institute of Psychiatry, King's College London and her NIHR Senior Investigator Award.

The PERCEIVE Team would also like to thank the NIHR MHRN who provided support for this project. 


\section{Conflict of Interest}

None

\section{References}

Addington, J., Saeedi, H. and Addington, D. (2006). Facial affect recognition: a mediator between cognitive and social functioning in psychosis? Schizophrenia Research, 85, 142-150.

Beck, A. T., Baruch, E., Balter, J. M., Steer, R. A. and Warman, D. M. (2004). A new instrument for measuring insight: the Beck Cognitive Insight Scale. Schizophrenia Research, 68, 319-329.

Cella, M., Swan, S., Medin, E., Reeder, C. and Wykes, T. (2013). Metacognitive awareness of cognitive problems in schizophrenia: exploring the role of symptoms and self-esteem. Psychological Medicine, 5, 1-8.

Combs, D. R., Adams, S. D., Penn, D. L., Roberts, D., Tiegreen, J. and Stem, P. (2007). Social cognition and interaction training (SCIT) for inpatients with schizophrenia spectrum disorders: preliminary findings. Schizophrenia Research, 91, 112-116.

Combs, D. R., Elerson, K., Penn, D. L., Tiegreen, J. A., Nelson, A., Ledet, S. N., et al. (2009). Stability and generalization of social cognition and interaction training (SCIT) for schizophrenia: six-month follow up results. Schizophrenia Research, 112, 196-197.

Combs, D. R., Penn, D. L., Wicher, M. and Waldheter, E. (2007). The ambiguous intentions hostility questionnaire (AIHQ): a new measure for evaluating hostile social-cognitive biases in paranoia. Cognitive Neuropsychiatry, 12, 128-143.

Corcoran, R., Mercer, G. and Frith, C. (1995). Schizophrenia, symptomatology, and social inference: investigating "theory of mind" in people with schizophrenia. Schizophrenia Research, 17, 5-13.

Couture, S. M., Penn, D. L. and Roberts, D. L. (2006). The functional significance of social cognition in schizophrenia: a review. Schizophrenia Bulletin, 32(suppl. 1), S44-S63.

Green, M. F., Oliver, B., Crawley, J. N., Penn, D. L. and Silverstein, S. (2005). Social cognition in schizophrenia: recommendations from the measurement and treatment research to improve cognition in schizophrenia new approaches conference. Schizophrenia Bulletin, 31, 882-887.

Gledhill, A., Lobban, F. and Sellwood, W. (1998). Group CBT for people with schizophrenia: a preliminary evaluation. Behavioural and Cognitive Psychotherapy, 26, 63-77.

Horan, W. P., Kern, R. S., Shokat-Fadai, K., Sergi, M. J., Wynn, J. K. and Green, M. F. (2009). Social cognitive skills training in schizophrenia: an initial efficacy study of stabilized outpatients. Schizophrenia Research, 107, 47-54.

Kay, S. R., Fiszbein, A. and Opler, L. A. (1987). The positive and negative syndrome scale (PANSS) for schizophrenia. Schizophrenia Bulletin, 13, 261-276.

Kern, R. S., Glynn, S. M., Horan, W. P. and Marder, S. R. (2009). Psychosocial treatments to promote functional recovery in schizophrenia. Schizophrenia Bulletin, 35, 347-361.

Kerr, S. L. and Neale, J. M. (1993). Emotion perception in schizophrenia: specific deficit or further evidence of generalized poor performance. Journal of Abnormal Psychology, 102, 312-318.

Martin, J. M., Warman, D. M. and Lysaker, P. H. (2010). Cognitive insight in non-psychiatric individuals and individuals with psychosis: an examination using the Beck Cognitive Insight Scale. Schizophrenia Research, 121, 39-45.

Moriarty, A., Jolley, S., Callanan, M. M. and Garety, P. (2012). Understanding reduced activity in psychosis: the roles of stigma and illness appraisals. Social Psychiatry and Psychiatric Epidemiology, $47,1685-1693$.

National Institute for Clinical Excellence (NICE) (2014). Psychosis and Schizophrenia in Adults: treatment and management, London: NICE. 
Parker, S., Foley, S., Walker, P. and Dark, F. (2013). Improving the social cognitive deficits of schizophrenia: a community trial of social cognition and interaction training (SCIT). Australasian Psychiatry, 21, 346-351.

Penn, D. L., Roberts, D. L., Combs, D. and Sterne, A. (2007). The development of the social cognition and interaction training program for schizophrenia spectrum disorders. Psychiatric Services, 58, 449451.

Penn, D., Roberts, D. L., Munt, E. D., Silverstein, E., Jones, N. and Sheitman, B. (2005). A pilot study of social cognition and interaction training (SCIT) for schizophrenia. Schizophrenia Research, $80,357-359$.

Pinkham, A. E., Penn, D. L., Green, M. F., Buck, B., Healey, K. and Harvey, P. D. (2014). The Social Cognition Psychometric Evaluation study: results of the expert survey and RAND panel. Schizophrenia Bulletin, 40, 813-823.

Roberts, D. L. (2007). Development and Preliminary Evaluation of a Social Cognition Intervention for outpatients with Schizophrenia Spectrum Disorders, Chapel Hill: ProQuest Information and Learning Company.

Roberts, D. L., Kleinlein, P. and Stevens, B. (2012). An alternative to generating alternative interpretations in social cognitive therapy for psychosis. Behavioural and Cognitive Psychotherapy, 40, 491-495.

Roberts, D. L. and Penn, D. L. (2009). Social cognition and interaction training (SCIT) for outpatients with schizophrenia: a preliminary study. Psychiatry Research, 166 (2-3), 141-147.

Roberts, D. L., Penn, D. L., Labate, D., Margolis, S. A. and Sterne, A. (2010). Transportability and feasibility of social cognition and interaction training (SCIT) in community settings. Behavioural and Cognitive Psychotherapy, 38, 35-47.

Roder, V., Mueller, D. R. and Schmidt, S. J. (2011). Effectiveness of integrated psychological therapy (IPT) for schizophrenia patients: a research update. Schizophrenia Bulletin, 37 (suppl. 2), S71-S79.

Schizophrenia Commission (2012). The Abandoned Illness: a report from the Schizophrenia Commission, London: Rethink Mental Illness.

Stain, H. J., Galletly, C. A., Clark, S., Wilson, J., Killen, E. A., Anthes, L., et al. (2012). Understanding the social costs of psychosis: the experience of adults affected by psychosis identified within the second Australian national survey of psychosis. Australian and New Zealand Journal of Psychiatry, 46, 879-889.

Tew, J., Ramon, S., Slade, M., Bird, V. and Le Boutillier, C. (2012). Social factors and recovery from mental health difficulties: a review of the evidence. British Journal of Social Work, 42, 443-460. 